\title{
Un caso de CIA compleja: ¿dónde están los bordes del septum interauricular?
}

\author{
Miguel Amor* \\ María Graciela Rousse** \\ Sergio Veloso***
}

\author{
Víctor Darú***** \\ Jorge A. Lowenstein ${ }^{* * * * *}$
}

\section{Correspondencia}

Miguel Amor

miguelamor68@gmail.com

\begin{abstract}
* Departamento de Cardiología. Investigaciones Médicas. Hospital Municipal de Agudos Ramos Mejía. Buenos Aires. Argentina
** Departamento de Cardiología. Investigaciones Médicas. Hospital Municipal de Agudos Carlos A Durand. Buenos Aires. Argentina

*** Departamento de Cardiología. Investigaciones Médicas. Hospital Universitario de Clínicas Gral. José de San Martín. Buenos Aires. Argentina

*** Departamento de Cardiología. Investigaciones Médicas. Sanatorio Finochietto. Buenos Aires. Argentina

***** Departamento de Cardiología. Investigaciones Médicas. Buenos Aires. Argentina
\end{abstract}

\author{
Recibido: 29/05/2020 \\ Aceptado: $12 / 06 / 2020$ \\ En línea: 31/07/2020
}

Citar como: Amor M, Rousse MG, Veloso S, Darú V, Lowenstein JA. Un caso de CIA compleja: ¿dónde están los bordes del septum interauricular? Rev Ecocar Pract (RETIC). 2020 (Jul); 3 (2): 39-41. doi: 10.37615/retic.v3n2a12

Cite this as: Amor M, Rousse MG, Veloso S, Darú V, Lowenstein JA. A complex ASD case: Where are the edges of the interatrial septum? Rev Ecocar Pract (RETIC). 2020 (Jul); 3 (2): 39-41. doi: 10.37615/retic.v3n2a12.

\section{Palabras clave \\ $\triangleright$ Defecto del tabique interauricular \\ $\triangleright$ Hipertensión pulmonar \\ $\triangleright$ CIA ostium secundum \\ $\triangleright$ Función diastólica \\ biventricular \\ $\triangleright$ Hiperflujo derecho}

Keywords

$\triangleright$ Atrial septal defect

$\triangleright$ Pulmonary hypertension

$\triangleright$ Atrial septal defect (ASD)

ostium secundum

$\triangleright$ Biventricular diastolic function

$\triangleright$ Increased pulmonary blood flow

\section{Presentación del caso}

Paciente varón de 45 años al que se detecta en un chequeo rutinario fibrilación auricular en el electrocardiograma de base. El paciente estaba completamente asintomático y realizaba de forma regular actividades normales de esfuerzo físico moderado a importante (levantar carga, cambio de neumáticos, futbol recreativo). Fue sometido a ecocardiografía transtorácica (ETT) donde se apreció dilatación de cavidades derechas y una comunicación interauricular (CIA) tipo ostium secundum de amplias dimensiones, por lo que el paciente fue referido a nuestro centro para la realización de una ecocardiografía transesofágica (ETE) para evaluar los bordes y descartar defectos asociados.

Se realizó una nueva ETT en la que se apreció un ventrículo izquierdo de tamaño y grosor normal, con fracción de eyección en límites normales y con movimiento septal paradójico (Figura 1A). La aurícula izquierda estaba moderadamente dilatada, $29 \mathrm{~cm}^{2}$ (Figura 1B). No había alteraciones valvulares de tipo estructural. Destacaba una dilatación severa de cavidades derechas con aurícula derecha con área de $42 \mathrm{~cm}^{2}$ (Figura 1B) y un ventrículo derecho (VD) con diámetros y longitud incrementados (Figura 1C). La función sistólica del VD está levemente deprimida con fracción de acortamiento de área del 30\%, excursión sistólica del anillo tricúspide de $19 \mathrm{~mm}$, onda $\mathrm{S}$ del Doppler tisular $(13 \mathrm{~cm} / \mathrm{s})$, índice de TEI de 0,49 (VN $\geq 0,55)$ y deterioro leve del strain longitudinal de la pared libre (-20\%) (Vídeo 1). Se observa un jet de insuficiencia tricúspide de pobre representación espectral, que no permite determinar la presión sistólica en arteria pulmonar (Figura 1D). Se estimó un gradiente ventriculoauricular de $35 \mathrm{mmHg}$, al que se suma $15 \mathrm{mmHg}$ de presión en aurícula derecha (por una cava inferior dilatada sin colapso), estimando una sistólica pulmonar de $50 \mathrm{mmHg}$. En el flujo del tracto de salida del VD no había acortamiento del tiempo de aceleración. Como se comentó, la vena cava inferior (VCI) estaba dilatada levemente $(23 \mathrm{~mm}$ ) con colapso inspiratorio menor al $50 \%$. Se observa un shunt de izquierda a derecha desde las ventanas apical y subcostal a través de un defecto amplio del septum interauricular (Vídeo 2) y se calculó un Qp/Qs de 5:1. 
En la ETE se detecta un gran defecto del septo interauricular con forma elíptica de $5 \times 3,5 \mathrm{~cm}$ medido con modo bidimensional; $y$ con un área de $14 \mathrm{~cm}^{2}$ en la reconstrucción tridimensional (Figura 2 y Vídeo 3). El defecto no tenía bordes adecuados para el procedimiento percutáneo con ausencia total del borde aórtico (Vídeo 4), esbozo (rodete) de borde inferior (Vídeo 5) y borde posterosuperior menor de $4 \mathrm{~mm}$. Existía un shunt amplio a través del defecto en el Doppler color (Vídeo 6). Se descartó la presencia de retorno venoso anómalo parcial y ductus, así como otras anomalías asociadas a nivel de la válvula mitral.

\section{Estudio por imagen}

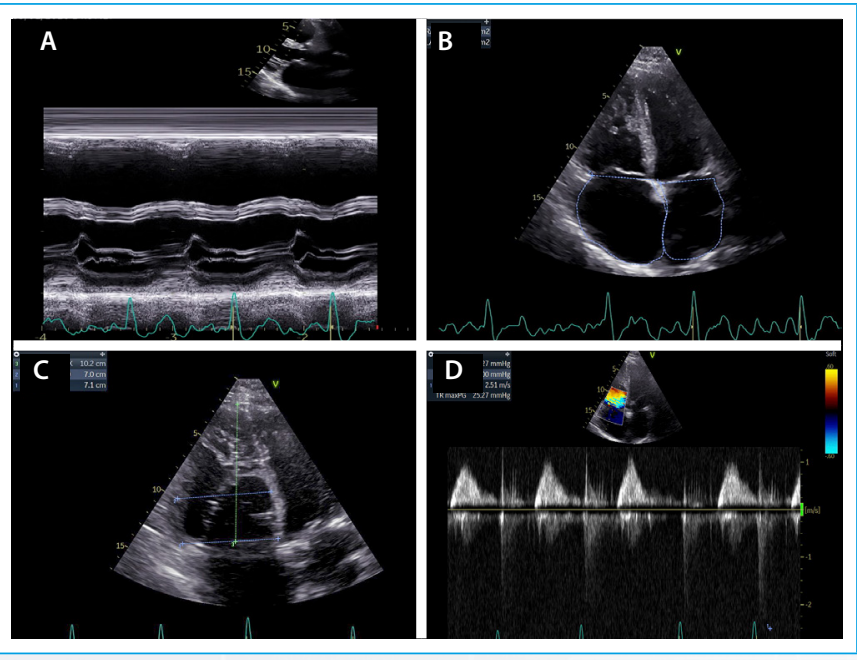

Figura 1. A: modo M que muestra dilatación del VD y septum paradójico; B: dilatación biatrial, severa; C: diámetros del VD en plano de cuatro cámaras; D: insuficiencia tricúspide leve en el Doppler continuo

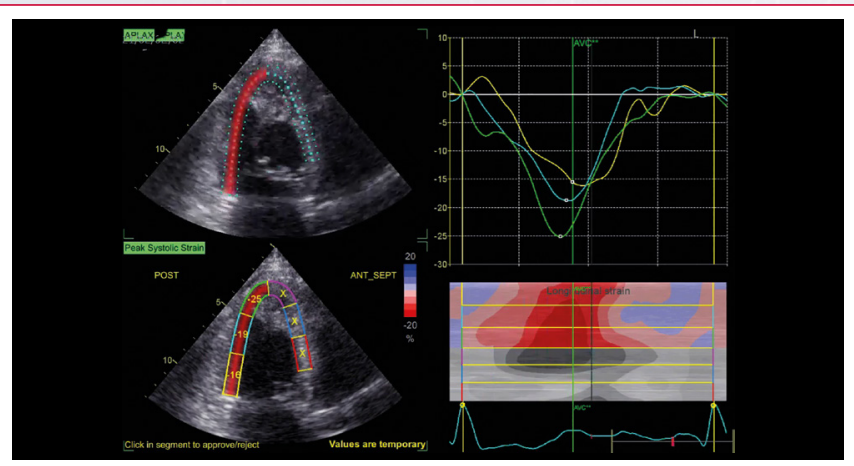

Vídeo 1. Strain de pared libre del VD en plano de cuatro cámaras

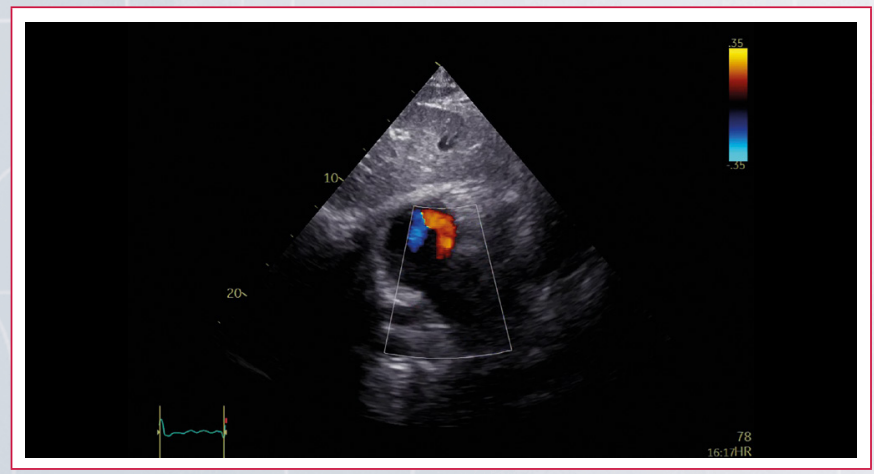

Vídeo 2. Imagen subcostal, flujo de Al a AD a través de CIA amplio con Doppler color

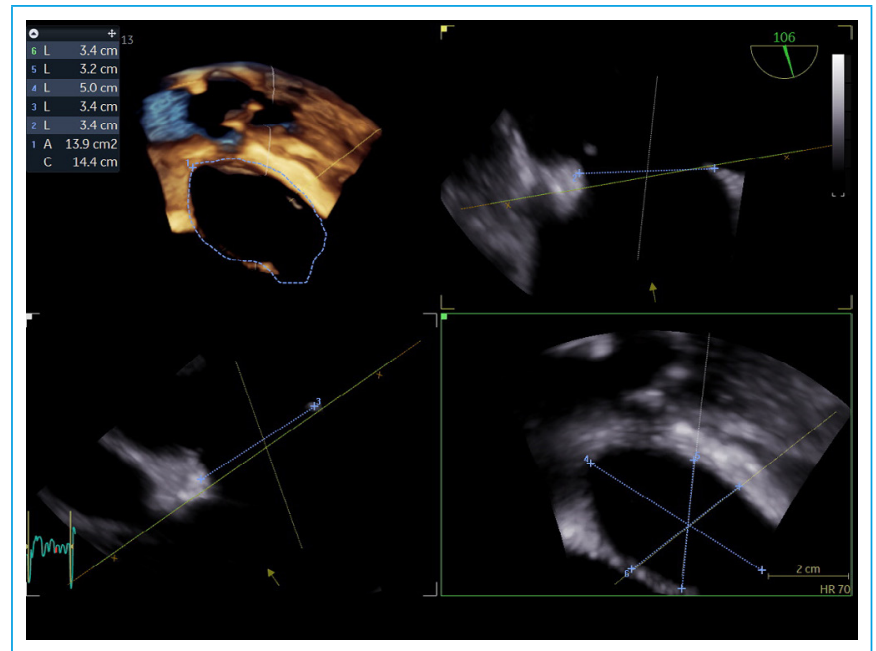

Figura 2. Reconstrucciones multiplanares a partir de la ETE tridimensional

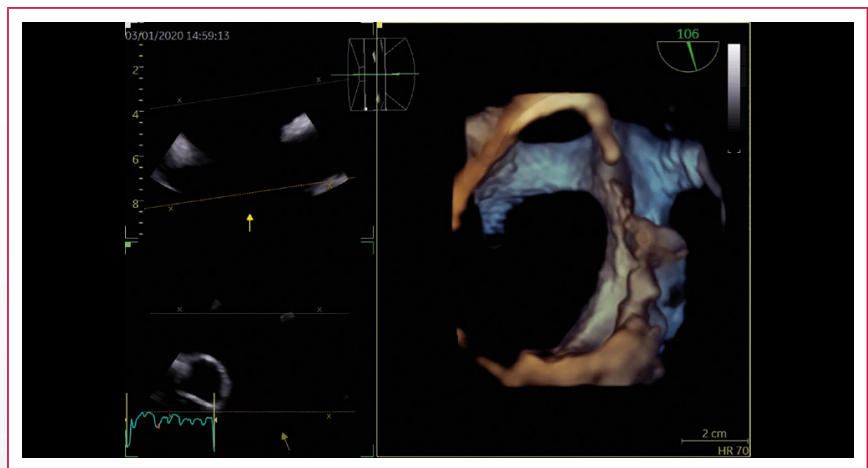

Vídeo 3. Reconstrucción tridimensional del amplio defecto del septo interauricular

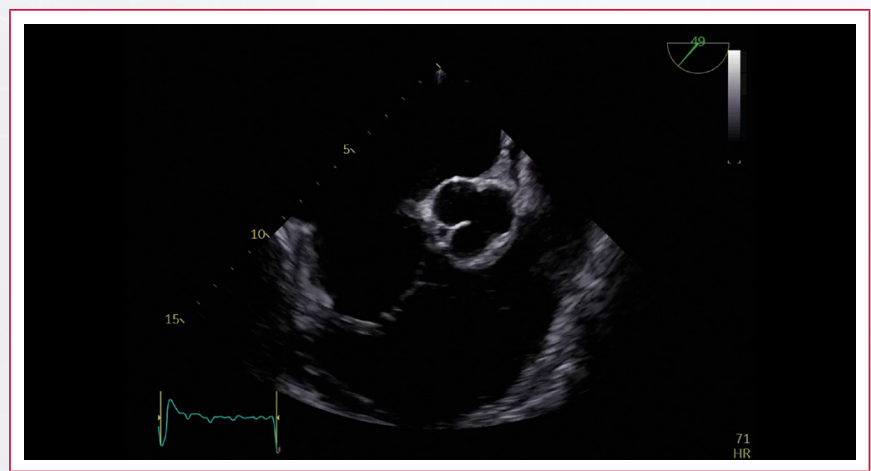

Vídeo 4. ETE, plano de $40^{\circ}$ que muestra ausencia total del borde aórtico

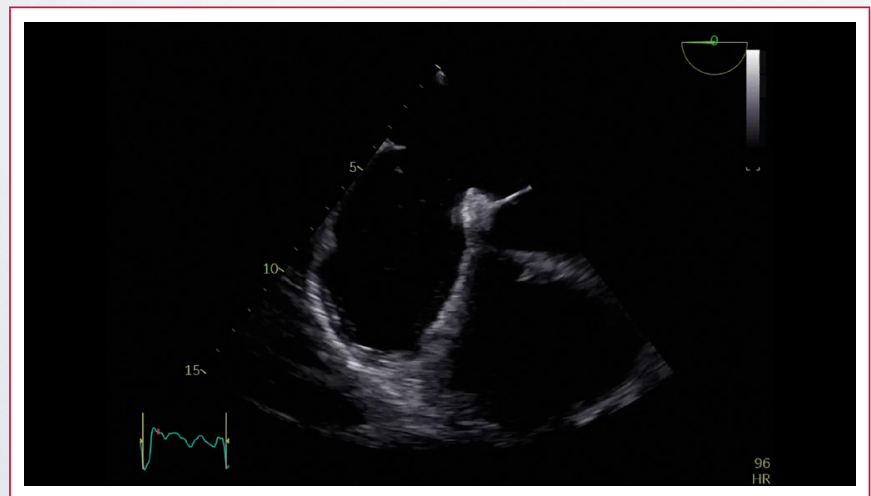

Vídeo 5. ETE, plano de $0^{\circ}$ que muestra borde inferior escaso 


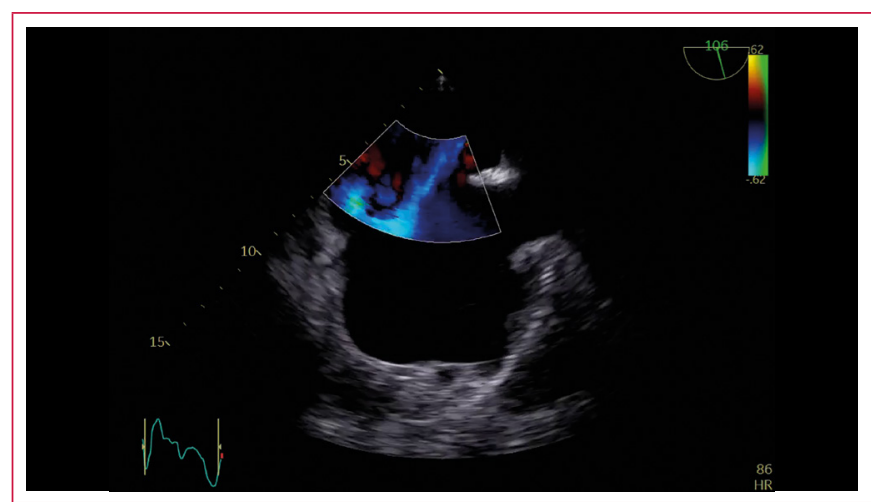

Vídeo 6. ETE plano a $106^{\circ}$ en vista bicava con Doppler color que muestra el shunt amplio a través del defecto

\section{Discusión}

Se trata del caso de un varón de 45 años con una CIA tipo ostium secundum, con un defecto amplio, curiosamente asintomático, sin signos aparentes de HTP y con función sistólica del VD levemente comprometida.

Es sabido que en una CIA grande se igualan las presiones entre ambas aurículas, y la distensibilidad de ambos ventrículos determinará el grado de hiperflujo. La HTP puede desarrollarse en respuesta a una sobrecarga crónica de la circulación pulmonar causada por una CIA de izquierda a derecha, con la consiguiente sobrecarga de volumen, aumento del gasto cardíaco derecho y una sobrecarga de la presiones pulmonares, lo que llevaría a un remodelado vascular pulmonar ${ }^{(1,2)}$. Pueden encontrarse diferentes valores de PSAP a pesar de similares sobrecargas de volumen. En un extremo del espectro hay adultos con enfermedad vascular pulmonar leve o nula y una derivación grande, y viceversa. También se sabe que sólo entre un 15\% y un 25\% de las CIA grandes desarrollan HTP, por lo que además del hiperflujo se postulan otros factores responsables del remodelado arteriolar, como por ejemplo genéticos, enfermedad vascular pulmonar, trombosis arterial pulmonar, anorexígenos, entre otros $^{(3,4)}$. Son también predictores de HTP, además del tamaño del defecto, la edad, la superficie corporal baja (SC) y el sexo femenino.

Las CIA de evolución crónica están asociadas con remodelado de la aurícula izquierda, caracterizado por el incremento de sus dimensiones (longitud, área y volumen), y con inestabilidad eléctrica que favorece la instalación de una fibrilación auricular. El comportamiento fisiopatológico va a ser diferente cuando se asocie:

- Una cardiopatía isquémica o hipertensiva con incremento del cortocircuito izquierda-derecha debido al aumento de la presión telediastólica del ventrículo izquierdo.

- Una HTAP que favorece la aparición de insuficiencia cardíaca derecha y al comprometer la función diastólica del ventrículo izquierdo se incremente el flujo venoso pulmonar que predispone a procesos infecciosos de las vías respiratorias, además de una limitación a la actividad física.

Se presentó cierta dificultad en clasificar esta CIA de enormes dimensiones, con ausencia de bordes aptos para su cierre percutáneo. No todas las CIA corresponden a un defecto septal. Las únicas que son verdaderos defectos septales son las comunicaciones tipo foramen oval, también conocidas como comunicaciones tipo ostium secundum. El verdadero septo auricular está constituido por la valva del foramen oval y la parte inferior del borde muscular. En el sector posterosuperior el borde corresponde a una invaginación de la pared de la aurícula, que también se conoce como septum secundum.
El sector anterosuperior se relaciona con la raíz aórtica que tiene una posición central en el corazón, profundamente acuñada entre las válvulas auriculoventriculares.

Se reconocen cuatro tipos de CIA. El más frecuente es el defecto a nivel del septo interauricular, es decir la CIA tipo foramen oval o también llamada ostium secundum. Las otras CIA son los defectos tipo seno venoso (superior e inferior), los defectos tipo seno coronario y los defectos tipo ostium primum, que tienen una unión auriculoventricular común como los demás defectos del septo atrioventricular ${ }^{(5,6,7)}$. En el paciente de este caso, el defecto es tan grande que podría considerarse que se ha extendido al seno venoso inferior ${ }^{(8)}$. Sin embargo, el hecho de tener un esbozo de borde posterosuperior descarta el diagnóstico de CIA tipo seno venoso de cava inferior y apoya al diagnóstico de CIA amplia tipo ostium secundum.

\section{Conclusión}

El paciente de este caso debe ir a cirugía para cierre con parche del defecto, ya que el abordaje percutáneo no es factible.

Se plantea la necesidad del estudio hemodinámico (cateterismo derecho, para determinar PSAP por este método) al fin de evitar subestimar el cálculo por Doppler de la presión pulmonar, sobre todo por la presencia de una función sistólica ventricular derecha levemente por debajo de los límites fisiológicos.

\section{Ideas para recordar}

- No siempre hay relación entre el tamaño del defecto y la PSAP.

- Medir todos los bordes es fundamental para la decisión de cierre percutáneo frente a cierre con parche.

- Siempre se deben descartar anomalías asociadas.

- Se debe comprobar siempre el valor de la presión pulmonar por cateterismo derecho, que permitirá también hacer el cálculo de las resistencias vasculares pulmonares.

\section{Bibliografía}

1. Lange, et al. Association between pulmonary hypertension and an atrial septal defect. Neth Heart J 2013; 21: 331-332.

2. Le Gloan $L$, et al. Patophysiology and natural history of atrial septal defect. Journal of Thoracic Disease 2018; 10 (Suppl 24): S 2854-S 2863.

3. Gabriels $C$, et al. A different vie won predictors of pulmonary hypertension in secundum atrial defect. International Journal of Cardiology 2014; 176: 833840.

4. Martin SS, et al. Atrial Septal Defects. Clinical manifestations, echo assesment and intervention. Clinical Medicine Insights: Cardiology 2014; 8 (S 1).

5. Anderson RH, et al. Development and structure of the atrial septum. Heart BMJ 2002; 88: 104-110.

6. Mori S, Anderson RH, et al. Demostration on living anatomy clarifies the morphology of interatrial communications. Heart BMJ 2018; 313-378.

7. Anderson RH, Brown NA, et al. Insights regarding the normal and abnormal formation of the atrial and ventricular septal structures. Clinical Anatomy 2016; 29: 290-304.

8. Snarr BS, liu MY, Zuckerberg JC, et al. The paraesternal short axis view improves diagnostic accuracy for inferior sinus venosus type of atrial septal defect by transthoracic echo. Journal of the American Society of Echocardiography 2017; 30 (3): 209-215. 